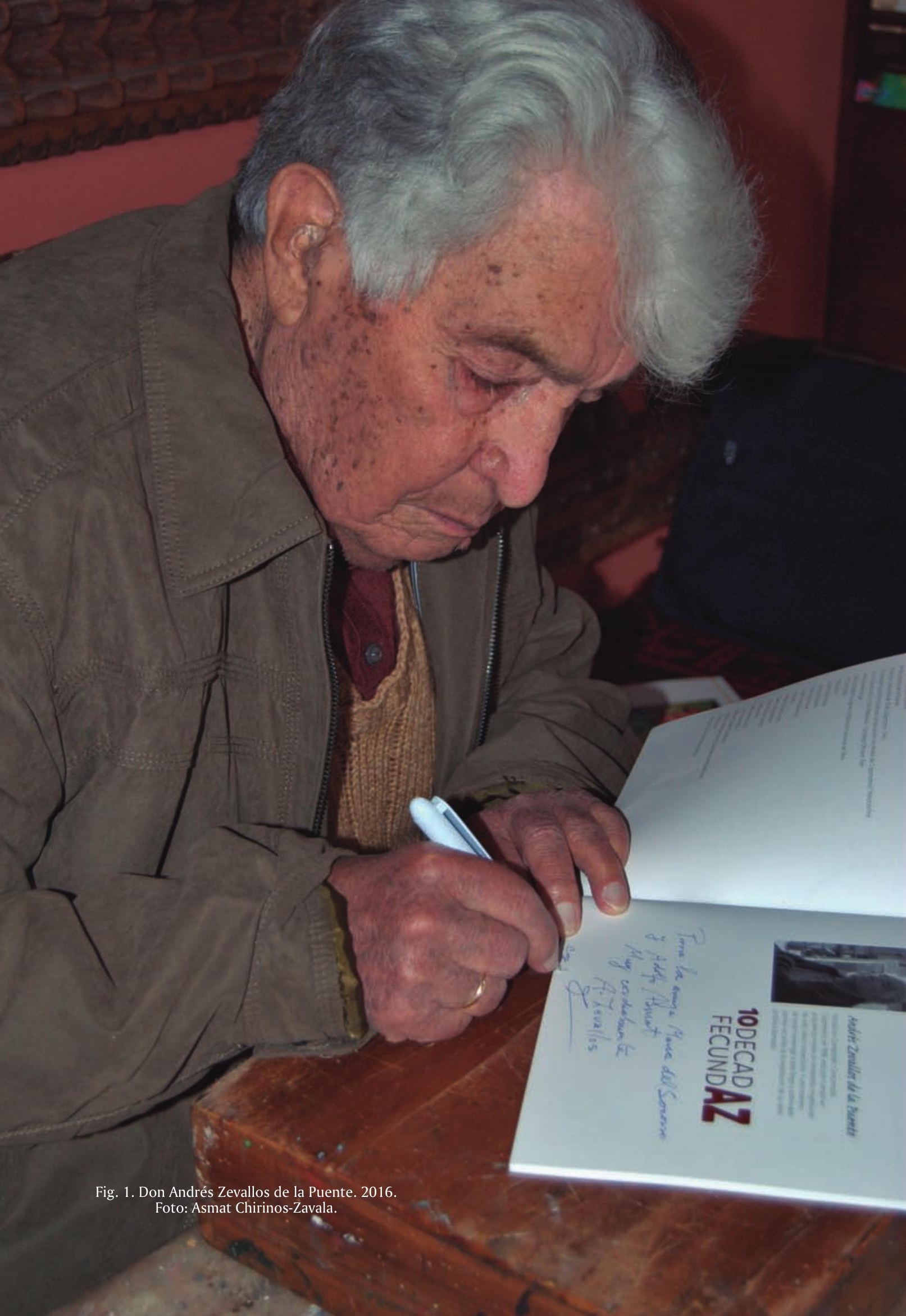




\title{
Andrés Zevallos de la Puente: aproximación neuroestética al artista y su obra
}

\author{
Dra. María del Socorro MoraC de Asmat \\ Asociación Española de Críticos de Arte \\ Asociación Internacional de Críticos de Arte \\ mdelsmorac@hotmail.com
}

Y no olvide quien aspire a separar Devoción y obligación

Que la vida aspira a la conciliación

Del mismo modo que ambos ojos

Convergen en la misma mirada.

Porque sólo cuando el amor y la necesidad sean uno

$Y$ el trabajo se convierta en un juego

Para este cuerpo mortal,

Ninguna acción quedará sin culminar

Para mayor gloria de los Cielos

$Y$ beneficio de las generaciones futuras.

Y una educación realmente sintética no puede conformarse con menos.

Robert Frost ${ }^{1}$

\section{Resumen}

Don Andrés Zevallos de la Puente fue un ser humano especial que buscó desarrollar al máximo sus capacidades. En este artículo analizaremos sus palabras desde el punto de vista psicobiológico y neuroestético. Comenzaremos por su infancia; sus inicios en el arte, descubriendo a los pintores que había en su tierra; sus estudios y avatares personales; su obra, que nos llevará directamente al amor que sentía por su tierra y costumbres, formando parte de la corriente indigenista, para terminar con sus reflexiones sobre la espiritualidad, la muerte y sus últimos consejos a las futuras generaciones. El recuerdo que nos queda de él es que supo conciliar devoción y obligación en su larga vida, con sabiduría y tesón.

Palabras clave: neuroestética, vida, obra, indigenismo, resiliencia, espiritualidad, memoria.

\begin{abstract}
Don Andrés Zevallos de la Puente was a special human being who sought to develop his abilities to the fullest. In this article we will analyze his words from a psychobiological and neuroesthetic point of view. We begin with his childhood, his beginnings in art discovering the painters who lived in his land, his studies and personal avatars, his work that will lead us directly to the love he felt for his land and customs, being part of the Indigenist current; to end with his reflections on spirituality, death and his last advice to future generations. The reminder we have of him is that he was able to reconcile devotion and obligation in his long life with wisdom and tenacity.
\end{abstract}

Keywords: neuroaesthetics, life, work, indigenism, resilience, spirituality, memory.

1 En: Root-Bernstein, R y M. (2002, p. 384). Para quien desee escuchar la versión en inglés: https://www.youtube. com/watch?v=oRosY_jyXfs 


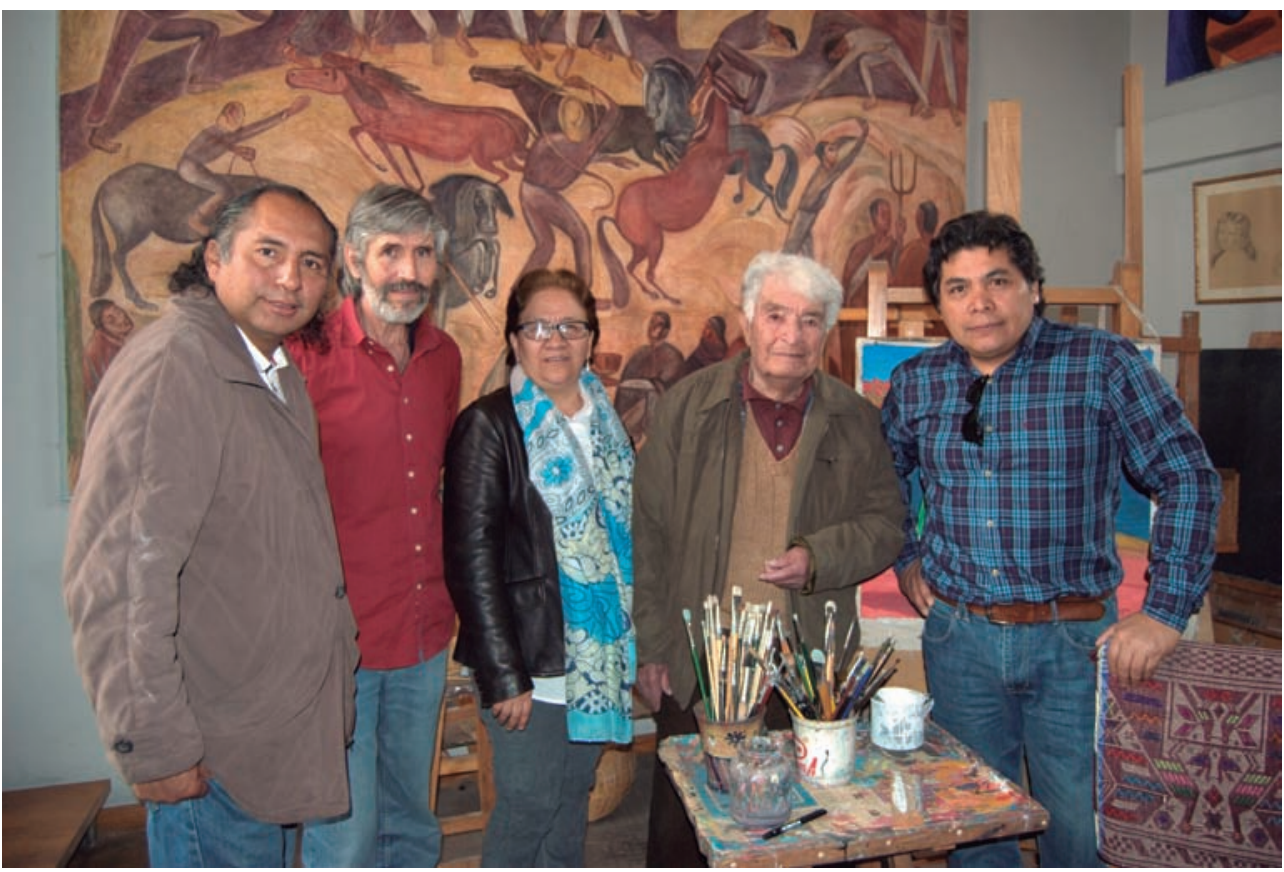

Fig. 2. En el taller de Don Andrés Zevallos de la Puente. Daniel Cotrina Rowe, Jorge Lombardi, Ma del Socorro MoraC y Asmat Chirinos-Zavala. 2016.

La neuroestética es una ciencia emergente (Cinzia \& Vittorio, 2009, pp. 682-687; MoraC., 2014, p. 69; MoraC, 2015, p. 68) que no solo "se ocupa exclusivamente del estudio de la belleza o preferencia, aunque estos conceptos son de importancia histórica, sino incluyen una amplia gama de emociones estéticas, juicios y comportamientos" (Marin, 2015, 9, 443). Esta manera de acercarnos a los comportamientos y productos estéticos "está ganando impulso" (Chaterjee, 2011, pp. 53-62) y creciendo rápidamente. La neuroestética es un gran desafío, no solo para la comunidad científica, sino para el público en general (Conway \& Rehding, 2013, p. 11), ya que "los correlatos neurales de la apreciación estética ahora están entrando en el foco de la atención" (Munar et al., 2012, p. 185). Esto quiere decir que el abanico de posibilidades se va ampliando, no solo gracias al avance de la ciencia, la tecnología o las nuevas hipótesis, sino al interés de aquellos que estamos intrigados con la vida y obra de aquellos que se dedican a producir belleza (Pearce et al., 2016, pp. 265-279).

Para nosotros, que un artista centenario como Don Andrés Zevallos de la Puente (fig. 1) nos concediera una entrevista ${ }^{2}$ para poder analizarla, ha sido una gran oportunidad en nuestra trayectoria profesional, y vamos a realizar esta tarea de reflexionar sobre sus palabras con las armas que nos brinda la neuroestética, contemplando una bella vida que ha producido hermosas obras, con la intervención de ciertas partes de nuestro cerebro, como nos explican los investigadores Jacobs, Renken \& Cornelissen (2012, p.7):

2 La entrevista se realizó el día 15 de diciembre del 2016, en el taller del maestro en Cajamarca, gracias a la gestión de Daniel Cotrina Rowe, Jorge Lombardi, Asmat Chirinos-Zavala (fig. 2) y José Luís Chávez. Desde el inicio de esta conversación, demostró -desde el punto neurobiológico- un excelente estado de ánimo y esa simpatía que nos da el buen humor, que muchos no conservan o quizá nunca han tenido. Recordemos que esta entrevista se realizó pocos días antes de su cumpleaños. Zevallos: casi 101 años; lúcido, inteligente, travieso, con una chispa vital que haría palidecer a muchos seres de menor edad. La juventud está en la mente, sin duda alguna. 
Las interacciones entre el tipo de juicio y el nivel de belleza apuntan a evaluaciones de belleza que dan por resultado más belleza. Un análisis de cerebro completo no destaca las regiones que muestran tales efectos de interacción. Los análisis de la región de interés sobre los grupos que aparecieron en los principales efectos de los juicios de belleza frente a la aspereza y los estímulos hermosos contra feos indicaron que muchas de estas regiones realmente muestran efectos de interacción entre estos dos contrastes. Esto significa que estos grupos pueden estar involucrados en evaluaciones de belleza. El giro fusiforme, la amígdala y la corteza frontomediana mostraron efectos de interacción. Para la amígdala y la corteza frontomediana, estas interacciones consistieron en respuestas más fuertes al nivel de belleza durante el juicio de la belleza. Este patrón es muy favorable para una participación directa en las evaluaciones estéticas.

En otras palabras, el juicio estético es un juego entre nuestra capacidad crítica, el miedo a lo desconocido o la sorpresa hacia lo imprevisto, y nuestra capacidad de aventurarnos hacia la contemplación de nuestro propio mundo interior. Esta entrevista -más una charla que una entrevista; dada la edad del maestro no debíamos cansarlo indagando largo tiempo- tuvo una aclaración inicial que nos hizo Don Andrés: "Tienes que tener en cuenta que soy un viejo 'recontraviejo'. Me fallan muchas facultades, sobre todo las intelectuales. La memoria ya no es la que fue. Me faltan las palabras y no puedo redondear mis ideas. A veces, no tengo manera de explicar lo que siento"3. iCuántos cambios debió pasar el cerebro de nuestro artista! Como dice José Viosca Ros (2017):

El cerebro no permanece inmutable a lo largo de la vida de una persona. Al contrario, está en continua transformación. Esta propiedad se denomina plasticidad y afecta a distintos niveles del cerebro, desde las sinapsis a las prolongaciones nerviosas de las neuronas o las regiones funcionales. (p. 37)

José Regidor García (2010), en un catálogo sobre arte y cerebro, nos brinda un escueto panorama sobre el envejecimiento neuronal:

Una vez alcanzada la madurez, en el cerebro se acelera progresivamente la muerte neuronal que puede alcanzar en la corteza cerebral humana una ratio de 1 neurona por segundo (lo que significa 85000 neuronas por día). Considerando que la cantidad total de neuronas en el cerebro es aproximadamente de $10^{11}$, podemos establecer que, en condiciones normales la actividad cerebral no debería verse gravemente afectada. Con el envejecimiento la capacidad de aprendizaje no se pierde si bien se enlentece, de la misma manera que se pierde paulatinamente la agilidad en determinados procesos mentales como es el caso de la memoria. Aún así, el cerebro del viejo sigue presentando sus características plásticas, de manera que sigue siendo capaz de establecer nuevas conexiones, pero sobre todo, necesita estar en pleno funcionamiento para mantener la arquitectura neural, constituida por todo el entramado conexional que conforma la red neural. En este sentido, más importante que la pérdida neuronal es la regresión del entramado de conexiones neurales la que va a caracterizar el envejecimiento cerebral y, en este caso, resulta evidente que en el cerebro "lo que no se usa, se pierde"; en otras palabras, mantener la actividad intelectual activa en todas sus facetas es premisa fundamental para un envejecimiento cerebral saludable. (p.24)

Frick \& Benoit (2010) han realizado estudios sobre ambientes enriquecidos que favorecen un buen envejecimiento. Y ese ambiente enriquecido, externo e interno, actuó a favor

3 Todas las referencias verbales que citemos del maestro proceden de la entrevista mencionada. 


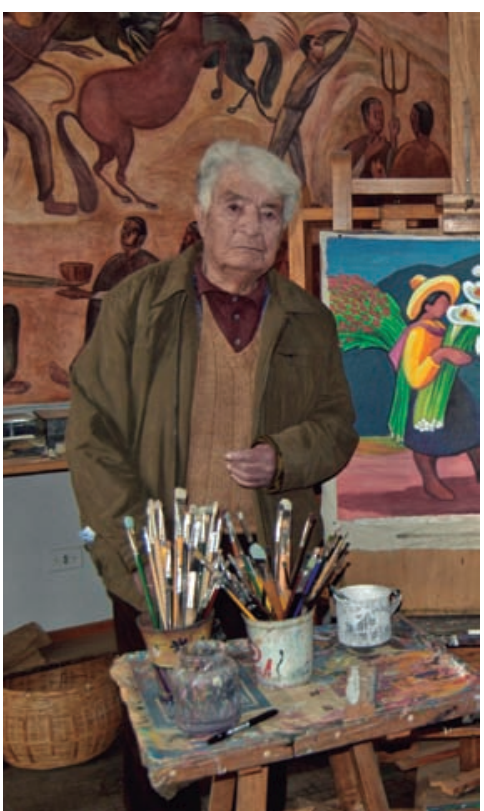

Fig. 3. Don Andrés Zevallos de la Puente. 2016.

Foto: Asmat Chirinos-Zavala.

del desarrollo de Zevallos ${ }^{4}$ (fig.3), un ser resiliente (Faye et al., 2017, p. 10), autotélico (Csikszentmihalyi, 2007, p. 146), constante, activo y productivo, como vemos en los principales logros con los que resumimos su carrera vital. Nace en Campodén (Leonardini, 2003, p. 194) el 30 de diciembre de 1916, pero luego se muda junto con su familia a Contumazá, un bello sitio de la sierra del Perú, muy cerca de Cajamarca. En esos verdes campos de un cielo despejado o de nubes tormentosas se desarrolló la infancia del maestro. "Todas las infancias son felices", dice muy optimista el maestro, sin reparar que hay seres humanos que desde muy temprana edad son llevados por las mareas del destino hacia la desgracia y aun hacia la muerte. Basta con ver las noticias de las olas migratorias en todo el mundo para reflexionar sobre las terribles circunstancias de algunas infancias. Pero, como decía el artista Brinkmann: "Lo bueno de la infancia es que no tenemos capacidad de comparación, por lo tanto, somos felices hasta que comenzamos a compararnos" ${ }^{5}$. Cuando se cambiaron a la casa del pueblo, su padre hizo una cosa inusual para los sitios en los que se vivía, más acostumbrados al empedrado: en el patio de la casa hizo un piso de cemento, en el cual él "comenzó a pergeñar figuras de animales, peces, paisajes”, fue así como se inició como artista, antes de ir a la escuela. También hacía figuras tridimensionales con arcilla (denominada mito, en su zona). En el colegio ya lo conocían y hacía los dibujos que le pedían. Todo lo que nos comenta el maestro nos remite a la fascinación que existe por conocer la infancia de los seres excepcionales, como escriben Kris \& Kurz (1982):

El interés universal por todo lo que se cuenta de la niñez y la juventud de las personas excepcionales está profundamente arraigado en la mente humana. Este interés se explica de dos maneras. Un punto de vista expresa que los acontecimientos de la infancia tienen una importancia decisiva para el futuro desarrollo del hombre, de aquí los intentos de demostrar la temprana influencia del destino en las vidas de los grandes personajes de la historia. El otro interpreta estas primeras informaciones sobre la vida de los héroes no como precursoras en cuanto a causalidad, sino como signos premonitorios; ve en las experiencias del niño un indicio de sus futuros logros y las considera como prueba de la temprana consumación de la singularidad. Esta opinión es más completa y es probable que sea la primitiva. (p. 31)

4 Don Andrés Zevallos de la Puente: escritor, maestro y renombrado pintor peruano, tuvo su formación artística signada por la influencia del indigenismo de José Sabogal, quién por entonces dirigía la Escuela Nacional de Bellas Artes. Es graduado en Educación por la Universidad Nacional de Cajamarca. Sus obras pictóricas se encuentran en colecciones peruanas como también en Alemania, Holanda, Bélgica, Francia, Inglaterra, Estados Unidos, entre otros países. Distinciones y condecoraciones: Orden del Sol Naciente, Rayos de Oro y Plata del Gobierno del Japón en mérito a su valiosa contribución al fortalecimiento de las relaciones entre nuestros países, en 2011. Medalla de la Región otorgada por el Gobierno Regional de Cajamarca en mérito a su despliegue de actividades en todos los campos culturales, sobre todo en lo referente al rescate, preservación y restauración del patrimonio artístico y monumental de la ciudad de Cajamarca. Hijo Predilecto del Colegio Nacional San Ramón de Cajamarca, en 2011. Profesor Honorario de la Universidad Ricardo Palma, en 2009. Doctor Honoris Causa por la Universidad Nacional de Cajamarca. Medalla de la PUCP por su exposición Cajamarca Tierra de Pintores en la Universidad.

5 Brinkmann, comunicación personal, octubre, 2012. 

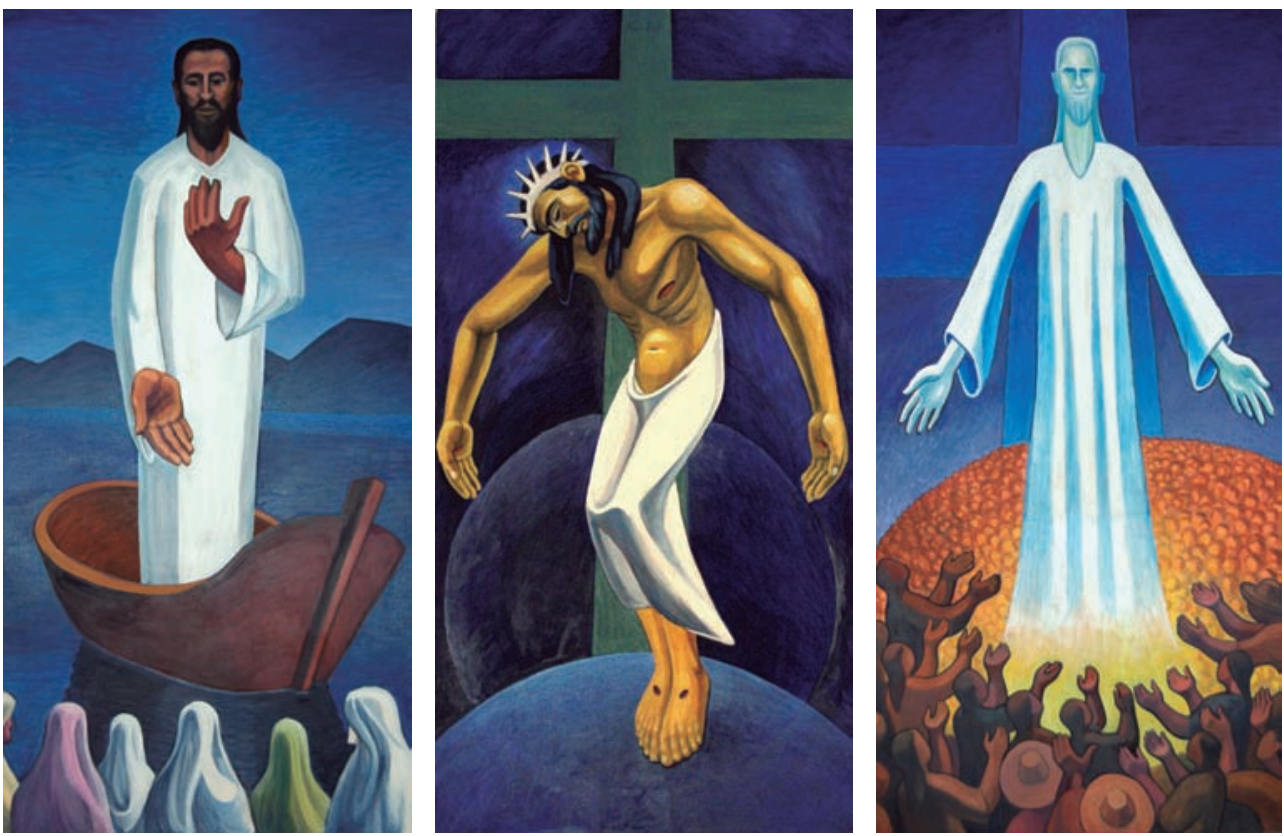

Fig. 4. Tríptico de los dos mil años. Técnica mixta sobre nordex. 2000.

Foto: Asmat Chirinos-Zavala.

El maestro se describe a sí mismo como una persona de temperamento tranquilo, pero muy inquieto con respecto a la naturaleza que lo rodeaba. Sus únicas amistades fueron los animalitos que encontraba en ese ambiente. Le gustaba también contemplar del río y los paisajes la naturaleza, pero no de una manera pasiva, sino indagando, buscando en su ambioma. Esto, según la teoría de la personalidad de Cloninger (Cloninger, 2004; Cloninger, 2007; Cloninger \& Zohar, 2011) y la teoría del SIC/SAC ${ }^{6}$ (Becerra, 2010; Aubi, Yousefi \& Alimoradi, 2011; Squillace, Picón \& Schmidt, 2011) denota una ambivalencia que no le llevó por caminos equivocados, al tener una gran capacidad de guardar solo los buenos sentimientos y apreciar todo lo que sus familiares hacían por él. Desde el punto de vista de Muntané (1994), un creativo tendría que tener un fondo oscuro, algún dolor, algún rencor que inconscientemente le impulsa a superarse. No es el caso de nuestro artista, quien cuenta que fue una persona muy cuidada por sus familiares (Syed \& Nemeroff, 2017, p. 10), en especial por su madre, quien le inspiró su lado religioso hacia el cual se rebeló más adelante, sin que ello le impidiese representar ciertos acontecimientos bíblicos (fig. 4), así como en su arte también está rescatado un hecho real, El rapto (fig. 5) de su bisabuelo Ramón por parte de su tatarabuela Ascencia. Buse (1993) comenta con respecto a este hecho:

No es común tener los mismos tatarabuelos por parte de padre y madre. Tampoco lo es pasarse como cincuenta años persiguiendo una quimera, aunque la vida, a cada rato, imponga todavía no. En la existencia de Andrés esas esperas fueron tantas que otros, menos tozudos, hubieran acabado por desistir. (p.5)

Su obra es el reflejo de su vida y su carácter, así como de su entorno privilegiado y particular: sus ancestros indígenas y españoles se mezclaron de manera muy singular en un espacio que él amó profundamente. Como comentamos en el catálogo realizado para la

6 Sistema de Inhibición y Activación Conductual. 


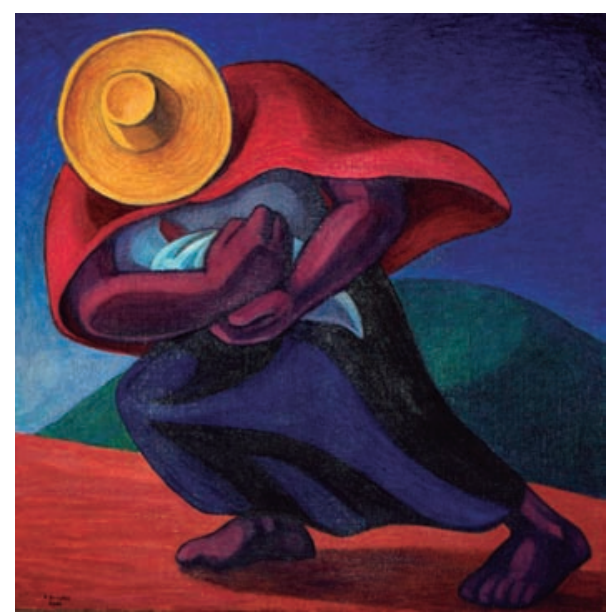

Fig. 5. El rapto. Técnica mixta sobre yute. 2002. Foto: Asmat Chirinos-Zavala.

muestra Sincretismo (MoraC., 2017, p. 8), al reflexionar sobre las palabras de Gazzaniga (2012), no somos cerebros aislados; somos nosotros, nuestro ambioma y nuestras circunstancias. Purves et al. (1997) resumen esta realidad diciendo: "La rica diversidad de personalidades, habilidades y conductas humanas es presumiblemente generada por la singularidad de los cerebros humanos individuales. Estas fascinantes diferencias neurobiológicas entre los humanos derivan de influencias hereditarias y ambientales" (p. 419).

Su única hermana, Antuca, era algo mayor y siempre se llevaron bien; no tuvo problemas familiares en su infancia. Le gustaba hacer cosas de muchachos, como mover las piedras, averiguar el nombre de las plantas, buscar animales, remover o transformar las cosas que encontraba a su alrededor, de modo que en su infancia se gestó ese gusto por la observación, sin que nadie le perturbara. Y todo ello lo fue guardando en su memoria, como lo dijo E. Román (2016): “(...) creo que sus pupilas comandaban sin fatiga un dibujo estrictamente para sí, algo que su memoria almacenaba prolijamente”(p. 7). Salas (2016) también escribió algo similar sobre los avatares del maestro: "La tromba del destino lo llevó por diversos caminos, para graduarse de hombre, pero su sensible retina -fiel a su acendrada vocación de pintor- lo ancló entrañablemente en el universo andino para captar peculiares perfiles humanos y constantes ajetreos cotidianos" (p. 9). Desde el punto de vista neuroestético, Zeki (2005) nos aclara el panorama del proceso cerebral que es, en el fondo, esa capacidad para observar, enfocar y registrar tan esencial para todo ser humano, en especial para los artistas visuales o plásticos:

Una de mis referencias favoritas dice que Monet "pintaba con los ojos, pero iSeñor, qué ojos!”. Esto, por supuesto, es una tontería: Monet, como muchos otros artistas pintaba con el cerebro y el ojo sólo actuaba como conducto que transmite señales visuales al cerebro. (...) Hace relativamente poco que nos hemos dado cuenta de que la imagen del mundo visual no se "imprime" en la retina del ojo, sino que ésta última es una mera etapa inicial de un proceso muy elaborado, diseñado para ver y que comienza en las denominadas "áreas superiores" del cerebro; la retina actúa como filtro esencial de las señales visuales y registra las transformaciones de intensidad de la luz, o la longitud de onda de la luz entre una parte de nuestro campo visual y otro, para después transmitir esas transformaciones registradas en la corteza cerebral. Aunque la anatomía de la retina es complicada, no contiene el mecanismo necesario para descartar la información innecesaria y seleccionar tan sólo aquella que necesitamos para representar los rasgos constantes y esenciales de los objetos. Gran parte de este mecanismo, de hecho, casi todo él, se encuentra en la corteza. (pp. 31-32) La elipsis es nuestra.

Aproximadamente con diez años, ya en Cajamarca, Don Andrés Zevallos se dio cuenta del valor de la pintura, porque conoció al pintor autodidacta Juan Villanueva Rodríguez, quien exhibía sus obras en su casa. Se hacía llamar Bagate y así firmaba sus obras. Bagate también fue un interesante fotógrafo (fig. 6), y hay una fecunda articulación de esa técnica a la elaboración de sus cuadros (Buntinx, 2003, pp. 42-43). Fue su profesor en la escuela, pero por situaciones reales de la vida, como hacer frente a los materiales y otros problemas, 
no pudo ser su profesor particular. Nuestro artista menciona que Cajamarca tuvo otros pintores muy importantes, con premios internacionales, como los tres pintores cajamarquinos que dan título al libro en el que el mismo maestro participó realizando los textos: Mario Urteaga, José Sabogal y Camilo Blas. En este libro, Zevallos (1991) reflexiona sobre el indigenismo, corriente artística a la cual perteneció:

Unos de los anhelos más caros para los indigenistas era el de realizar una especie de inventario paisajísticocultural del territorio nacional. Todos los miembros del grupo, unos más y otros menos, se dieron a explorarlo con el propósito de dar con la entrañable relación hombre-paisaje, admitiendo de esta manera el rechazado mote de indigenistas, pues Sabogal definió este propósito de forma muy clara diciendo: “...somos indigenistas en el justo significado

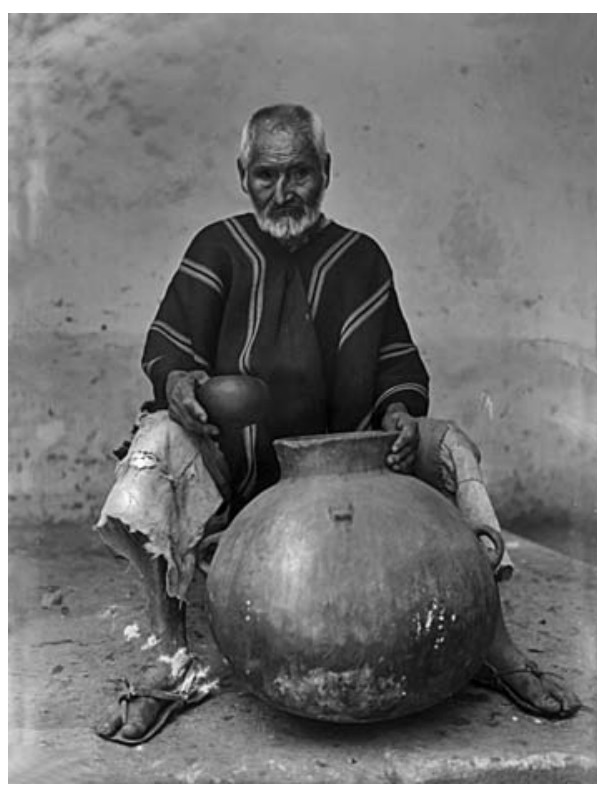

Fig. 6. El chichero.

Copia de gelatina de plata sobre papel. 1935. Foto: Juan del Carmen Villanueva Rodríguez Bagate. de la palabra y más aun, indigenistas culturales, pues buscamos nuestra identidad integral con nuestro suelo, su humanidad y nuestro tiempo. No admitimos aquello de raza superior aria en nuestro medio, pero sí estamos convencidos que en América ha surgido un hombre nuevo". (p. 14)

El maestro nos relata que no era mal alumno, pero que el arte y la literatura era lo que más le apasionaba. Como dice Zeki (2009), hay ciertas cosas, como el amor, que van más allá de la elección consciente, lo mismo pasa con la vocación ${ }^{7}$. A su familia no le gustó su elección, pero fueron consecuentes y le dejaron seguir su camino. Su madre hubiese preferido que fuese cura $^{8}$, pero él ya se había desengañado de $\operatorname{los} \operatorname{dog} a^{9}{ }^{9}$, aun cuando desde pequeño fue monaguillo, luego acólito y ayudaba ya en las tareas de la iglesia. Cuando su madre le regañaba por su falta de fe y vocación religiosa, siendo ella misma la que le había guiado y enseñado, él respondía inocentemente: "Sí, madre... me enseñaste a rezar, pero también a leer”. Fue a través de la lectura que amplió su mundo conceptual y su consciencia, algo que al parecer tienen en común los grandes artistas, como Vincent van Gogh, que en sus cartas $^{10}$ menciona los libros que ávidamente iba leyendo. Y nos dijo, sonriente: "Y de leer y de aclarar tantas preguntas que nos hace la vida, tengo yo mis ideas y hay cosas que de la religión no acepto. Como, por ejemplo, lo que nos enseña el Padrenuestro. ¿Quién ha visto lo que nos dice esa oración? Rezar por obligación ya no es posible en estos tiempos". Consciente de la dificultad del camino elegido, pensó no tener nunca compromisos: ni familia, ni hijos. Pero la vida le dio dos familias y ocho hijos, por lo que su carrera artística

7 Zeki, en su estudio sobre el amor, opina: Free will is restricted, even in love, pp 26-27.

8 Fontan del Junco (1996) dice: "La identidad del artista contemporáneo es una identidad sacerdotal", con lo que podemos deducir que la disciplina aprendida de niño fue trasladada al campo del arte y la cultura (p. 36).

9 Según Harari (2011, p. 234), "La religión puede definirse así como un sistema de normas y valores humanos que se basa en una creencia en un orden sobrehumano y aunque es un gran unificador de la humanidad, no puede aún examinarse el tema científicamente" (Harari, 2015, p. 134).

10 Gogh, V. van (2004) Cartas a Theo. 


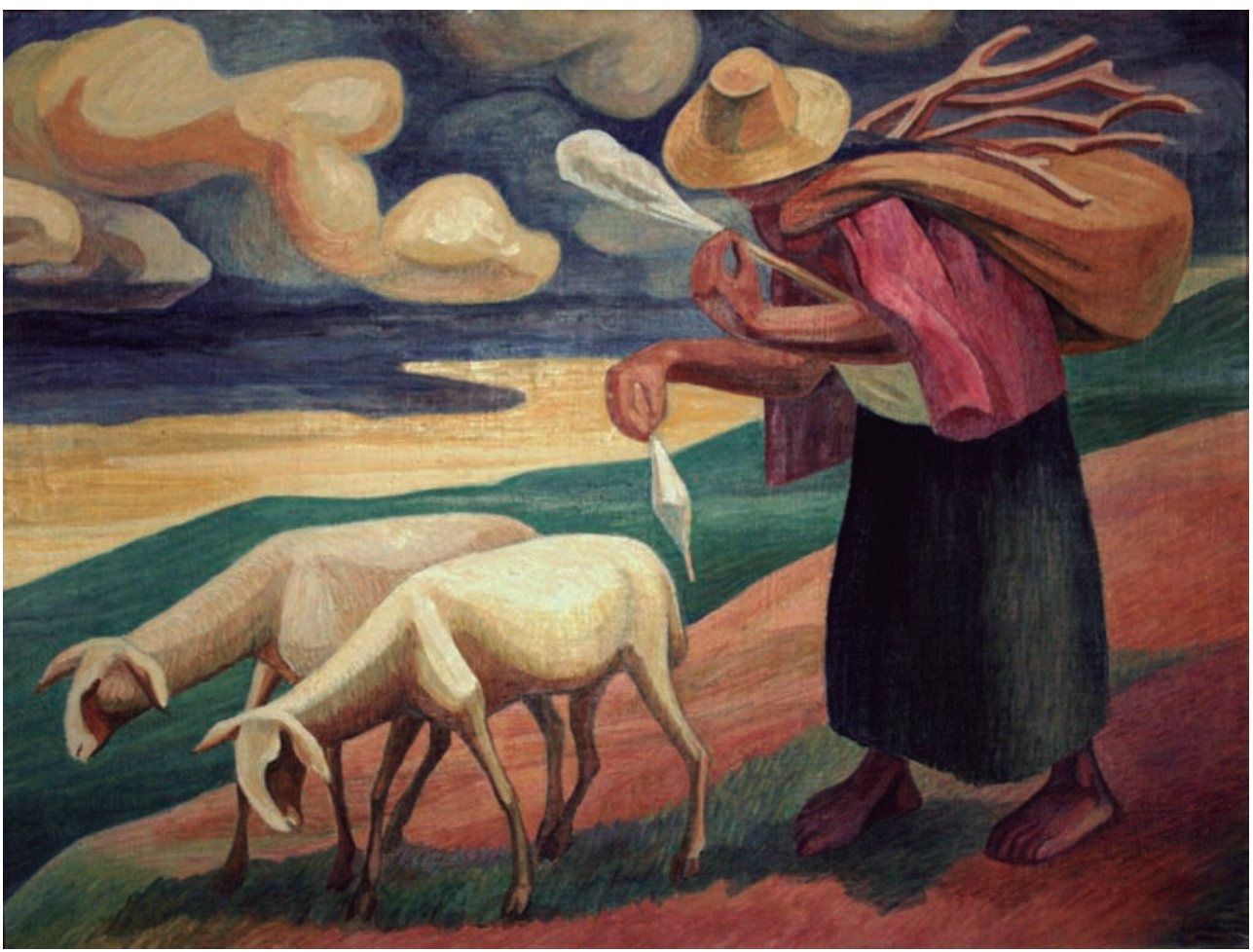

Fig. 7. El retorno. Óleo sobre yute. 1986.

Foto: Asmat Chirinos-Zavala.

fue diferente y singular. Nos dice que ha preferido ir solo, ser fiel a sí mismo, evitando los jurados o concursos. Su comportamiento y capacidad de postergar sus metas sin olvidarlas revela un enfoque empático hacia su entorno ${ }^{11}$ y un férreo autocontrol, que dependen de muchos circuitos cerebrales y que sin duda estaban muy bien interconectados:

Un componente clave para la toma de decisiones racional y los comportamientos apropiados dirigidos a metas es la capacidad de evaluar el valor de recompensa, la previsibilidad y el riesgo con precisión. El circuito de recompensas, central para la mediación de esta información y para evaluar los resultados probables de diferentes opciones con eficacia, es una compleja red neuronal. Mientras que el hipotálamo y otras estructuras subcorticales están involucrados en el procesamiento de información acerca de las recompensas básicas o primarias, las estructuras corticales y subcorticales del cerebro anterior se involucran cuando se requieren opciones complejas sobre estas necesidades fundamentales. Además, las opciones suelen incluir recompensas secundarias, como el dinero, el poder o el reto, que son más abstractas (en comparación con las necesidades primarias). Aunque las células que responden a diferentes aspectos de la recompensa como la anticipación, el valor, etc., se encuentran en todo el cerebro, el

11 Según Goleman (2013): “La empatía depende del músculo de la atención ya que, para sintonizar con los sentimientos ajenos, es preciso conectar con los signos faciales y vocales y otros indicios de sus emociones. La región cingulada anterior, una parte de la red de la atención, nos permite conocer la ansiedad de los demás movilizando nuestra amígdala, que resuena con esa ansiedad. En este sentido, la empatía emocional se 'encarna', porque nos permite sentir, en nuestro cuerpo, lo que está ocurriendo en el cuerpo de otra persona”. (pp. 130-131). 
centro de esta red está en el circuito cortical-ventral de los ganglios basales. Esto incluye la corteza orbitofrontal y la corteza cingulada anterior, el estriado ventral, el pallidum ventral y las neuronas de dopamina del cerebro medio. En general, los ganglios basales trabajan en conjunto con la corteza para ejecutar comportamientos motivados y bien planificados. El circuito de recompensa está integrado en este sistema y es una fuerza impulsora clave para el desarrollo y monitoreo de estos comportamientos. Otros circuitos de los ganglios basales, los asociados con la cognición y el control motor trabajan en tándem con elementos del sistema de recompensa para desarrollar acciones dirigidas a objetivos apropiados. (Harber, 2011)

Ya en la capital, Lima, se encontró con la efervescencia del indigenismo. El director de la Escuela de Bellas Artes, Sabogal, tenía "ideas drásticas para implantar un arte peruano". La maestra que guio su aprendizaje fue Teresa Carvalho, quien tuvo mucho interés por su desarrollo artístico. La escuela, más que el oficio, afinó en él el profundo sentimiento que ya albergaba por su tierra. Zevallos tuvo mucho interés en la raza autóctona, sus paisajes, sus costumbres (fig. 7). Lo que nos dijo en la entrevista coincide con lo que escribe Bazán Zurita (2016):

Dice Luis Eduardo Wuffarden: "si bien la primera batalla a favor del indigenismo fue dada en Lima, Sabogal instó a varios de sus discípulos provincianos a volver hacia sus lugares de origen y propagar allí sus principios programáticos. Es el caso de Andrés Zevallos, uno de los últimos continuadores directos de la escuela sabogalina”. Y, el mismo don Andrés, añade sobre esta etapa: "más que el aprendizaje de ciertas técnicas, mi paso por la escuela significó el encuentro de un camino hacia la comprensión de mi país, del mundo del que procedía, cuya verdad y belleza solo puede encontrarse por el conocimiento y disfrute profundo de su autenticidad". (p. 5)

Alfonso Castrillón Vizcarra, en su libro Tensiones generacionales (2014), se plantea esta pregunta: “¿Cuál es el carácter de la pintura en el Perú?” Y responde: "Para responder a esta pregunta no creo necesario, en esta oportunidad, remontarme hasta la Colonia, pero sí volver nuestros pasos hasta un capítulo que no puede dejarse de lado en la Historia del Arte Peruano; me refiero al Indigenismo” (pp. 27-28). Stastny (1967), sobre esta corriente, dice: "Una renovación total se producirá en el ambiente artístico peruano con la llegada de José Sabogal (1888-1956) a la dirección de la Escuela de Bellas Artes, en 1933” (p. 55). Bákula Budge (2004) escribe:

El efecto de esa propuesta, audaz y contestataria rindió sus frutos. El arte de nuestras corrientes nacionales, aún en sus versiones y tendencias más vanguardistas, se nutre y recurre a las fuentes estéticas de los fundamentos de las culturas indígenas. No es posible entender ni acercarse a la pintura contemporánea si no la entendemos como nacida de las vertientes del indigenismo, entendido como el deseo por conocer y revalorar lo propio, dotándolo del valor y dignidad que merecen. (p.43)

Zevallos, como hombre resiliente ${ }^{12}$ y autorrealizado (Maslow, 2003, p. 174) que fue, desarrolló un indigenismo muy particular, emparentado con las sólidas figuras del muralismo mexicano, ciertos toques naif y un posmodernismo neoperuano ligado a los fauves o a la antropofagia de Tarsila do Amaral (fig. 8), por la simplificación de la figura, los contrastes

12 Rojas Marcos (2011) nos aclara lo que se necesita para ser resiliente: "Los seis pilares de la resiliencia son elementos distintos que se complementan entre sí, empezando por las conexiones afectivas gratificantes con otras personas y ciertas funciones ejecutivas personales, como la aptitud para regular las emociones, identificar metas y programar pasos para conseguirlas. También figuran entre ellos la localización del centro de control en uno mismo, en lugar de desplazarlo a fuerzas externas, y la autoestima saludable. Los otros pilares son la tendencia a percibir y explicar las cosas positivamente o considerando sus aspectos más favorables, y la conciencia de motivos personales que den significado a la propia vida". (p. 63) 


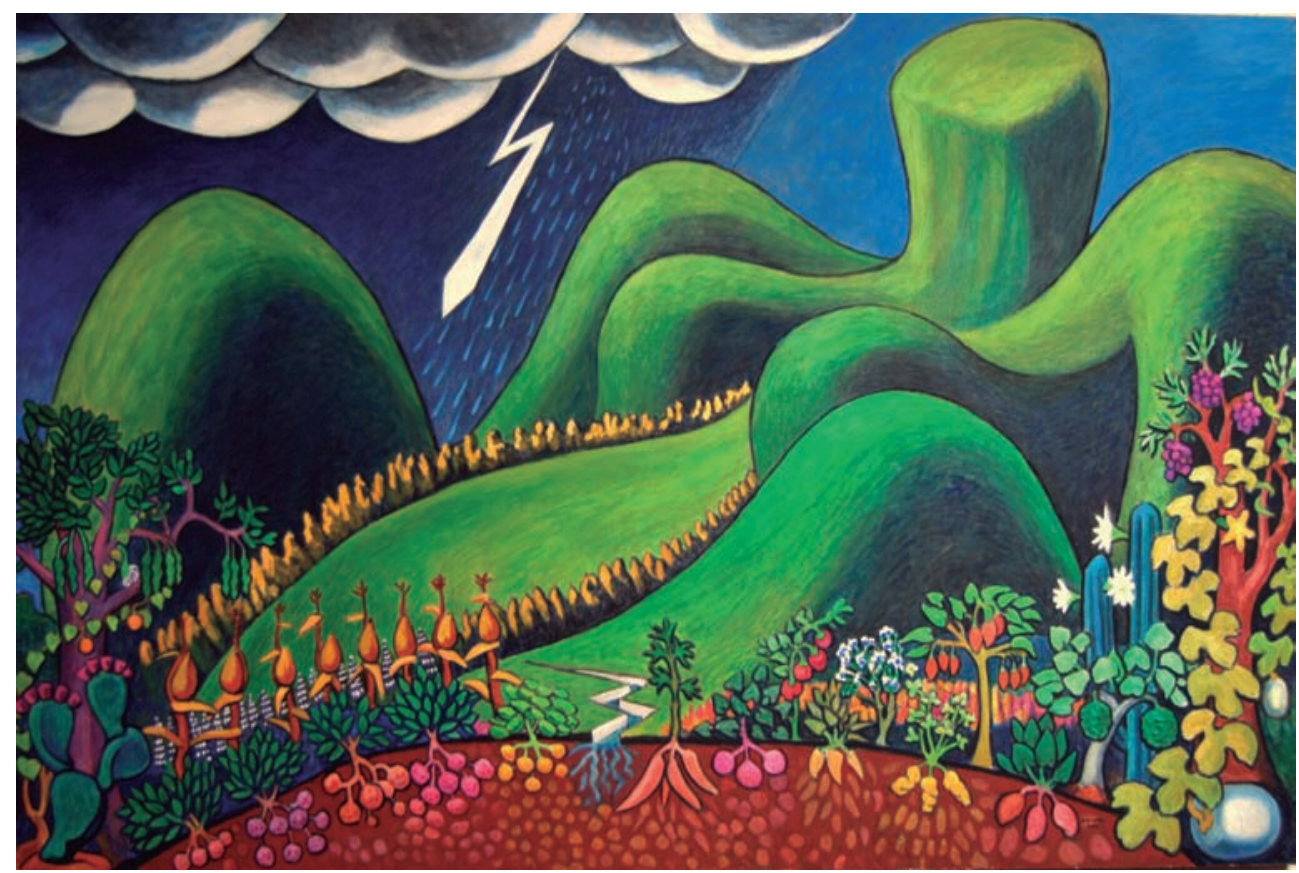

Fig. 8. Pachamama. Técnica mixta sobre nordex. 2002. Foto: Asmat Chirinos-Zavala.

y el colorido, que también están emparentados con la multiplicidad de tonalidades en los tejidos andinos. Pese a no ser un hombre religioso, en el sentido de creer en otros mundos o dimensiones ${ }^{13}$, tenía su propio mundo sagrado, el mundo del arte que no permitió que se contamine con prebendas ni concursos. Se mantuvo apartado de muchos de circuitos, será por ello por lo que no ha sido mencionado en muchas de las fuentes que hemos revisado. Podemos catalogarlo como a una persona que ha sabido emplear su tiempo, encaminar sus energías y llegar a la vejez con alegría y sabiduría, como una bella persona que no dejó de buscar la belleza (Conway \& Rehding, 2013, p. 11) en todo lo que hacía, ya que su compromiso con el arte y la cultura fue amplio. Cotrina (2016) escribe en el catálogo de su última exposición: "Sus obras se exponen en uno de los monumentos emblemáticos del barroco cajamarquino del siglo XVIII, el Conjunto Monumental de Belén, que fue conservado y restaurado durante su gestión como director de la Casa de la Cultura" (p. 13), lo que nos muestra de su buen hacer como gestor. Sin embargo, pocos podemos imaginar el inmenso esfuerzo que dedicó y todo lo que tuvo que dejar para seguir su camino. La subjetividad esencial de su experiencia, o qualia (Denton, 2009, p. 283), algo intrínsecamente personal, estuvo guiada, sin duda, por su sinosia ${ }^{14}$, no muy usual en esta era de especialistas o gente cerrada que no comprende que todo está interconectado.

13 No nos contestó con un "sí" o un "no" sobre la vida después de la vida, sino con un pensamiento de una lápida romana: "No era y fui/ Fui y no soy/ Vosotros que vivís/ Comed, bebed y regocijaos/Todo lo demás es mentira".

14 Root- Bernstein, R \& M. (2002) nos aclaran este término: "El reconocimiento de la naturaleza sintética de la comprensión creativa es tan escaso que ni siquiera existe palabra alguna para referirse a ella. Es por este motivo por lo que nosotros sugerimos el término sinosia, un término compuesto por el prefijo griego syn (que significa unión, como ocurre con el vocablo síntesis) y la palabra gnosis (el conocimiento) o noesis (que significa ejercicio de la razón o de la cognición). Así pues, el término sinosis o sinosia hace referencia al conocimiento sintético, a la unión de modalidades diferentes del conocimiento" (p. 361). 


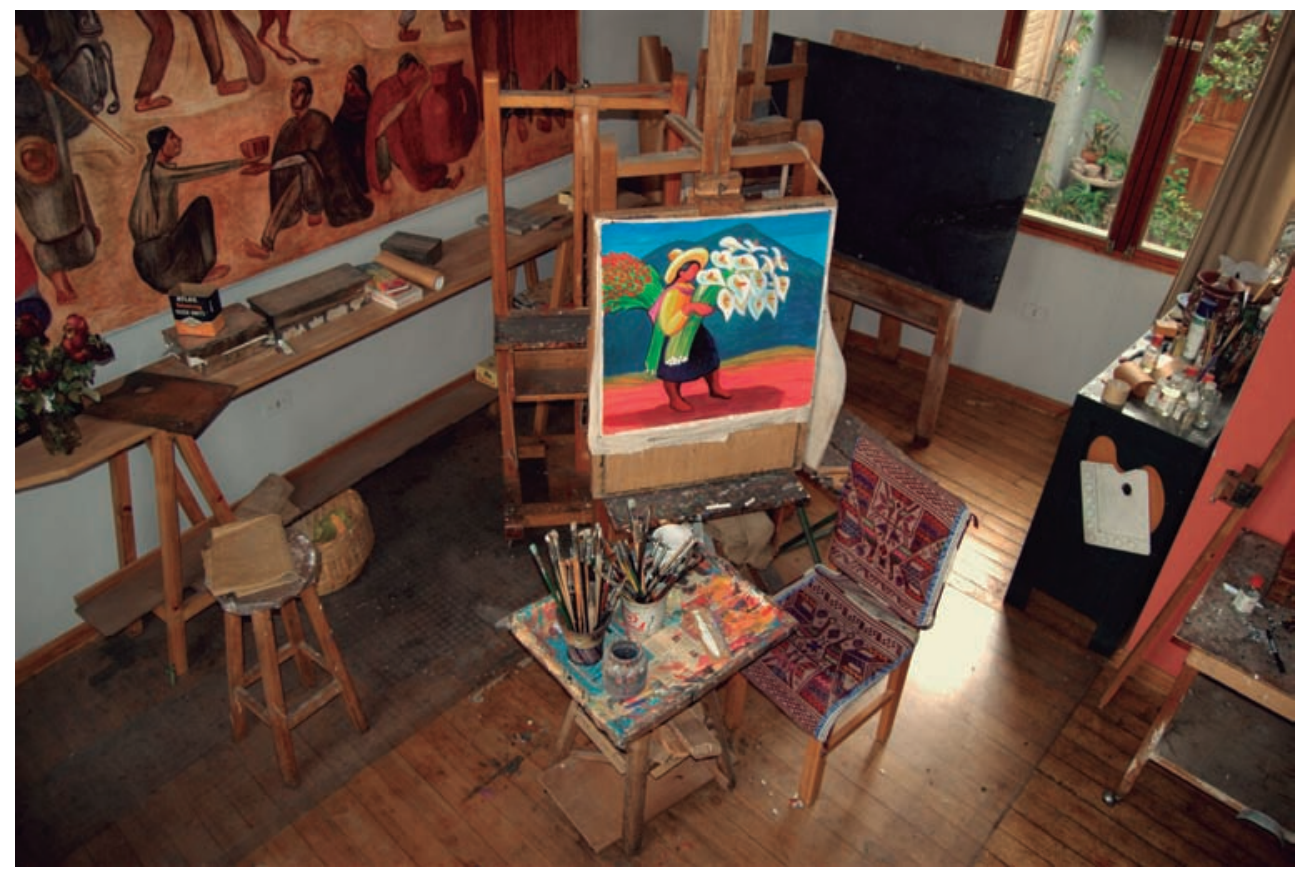

Fig. 9. Taller de maestro Andrés Zevallos de la Puente, en Cajamarca 2016. Foto: Asmat Chirinos-Zavala.

Zevallos de la Puente, como escribe Élida Roman (2005-2006), es un "caso singular, ejemplar y especialmente exitoso (...), cajamarquino por tradición y convicción"15 (p. 2). Las palabras que nos brindó para los futuros artistas revelan mucho de su temperamento y carácter: "Yo no creo en la inspiración. Creo en la transpiración. Cuando uno trabaja y trabaja con honestidad y resuelve sus problemas estéticos, materiales, espirituales o de otra índole, al final obtiene los frutos. Pero hay muchachitos que se creen artistas, que hacen un garabato y ya se creen Picasso. iEso no puede ser!". Nos despedimos de este ser humano tan completo y digno de estudios como el de Faye et al. (2017), sintiendo que nos acompañará, aun en su ausencia ${ }^{16}$ (fig. 9), como dice Gibran (2010): "Viviré más allá de la muerte y cantaré en vuestros oídos, / Incluso después que el inmenso mar me devuelva/a la profundidad del inmenso mar./Me sentaré a vuestra mesa, aunque sin cuerpo,/e iré con vosotros a vuestros campos como un espíritu invisible" (p. 214).

15 La elipsis es nuestra.

16 El maestro murió el viernes 7 de abril del 2017 en Cajamarca. 


\section{Referencias bibliográficas}

Aubi, S., Yousefi, S. \& Alimoradi, L. (2011). Relationship Between Brain/Behavioural Systems and Mental Health Among Students. ProcediaSocial and Behavioral Sciences (Vol. 30, pp. 16831687). Recuperado de http://www.sciencedirect. com/science/article/pii/S1877042811021501

Bakula, C. (2004). "El Indigenismo en la pintura peruana”. En Huertas Muñoz (comisario) Pintura Peruana Contemporánea (pp. 37-43) Catálogo de exposición realizada en Córdoba en la Sala de Exposiciones Museísticas Caja Sur, del 11 de mayo al 5 de junio. Córdoba: Cajasur.

Bazán, H. (2016). 10 Décadas Fecundas. En Cotrina, León, Vigo \& Armas (Producción). 10 Décadas Fecundas. Andrés Zevallos de la Puente (pp. 4-5). Catálogo de exposición en el Conjunto Monumental de Belén, Cajamarca.

Becerra, J. A. (2010). "Actividad de los sistemas de activación e inhibición conductual y psicopatología". Anuario de Psicología Clínica y de la Salud (6: 61-65).

Buntix, G. y Wuffarden, L. E. (2003). Mario Urteaga. Nuevas miradas. Lima: Museo de Arte de Lima \& Fundación Telefónica.

Buse, M. (1993). Claroscuro. Cajamarca: Ed. Martínez Compañón.

Castrillón, A. (2014). Tensiones generacionales. Lima: Editorial Universitaria URP.

Cinzia, D. D. \& Vittorio, G. (2009). "Neuroaesthetics: a review". Current opinion in neurobiology. (19 (6): 682-7). Recuperado de https://www.ncbi. nlm.nih.gov/pubmed/19828312

Cloninger, C. R. (2004). Feeling Good. The Science of Well-Being. New York: Oxford University Press.

Cloninger, R. C. (2007). "Genética”. En Oldham, J. M.; Skodal, A. E. y Bender, D. S. Tratado de los trastornos de la personalidad (pp. 145-155). Barcelona: Elsevier Masson.

Cloninger, C. R. \& Zohar, A. H. (2011). "Personality and the perception of health and happiness". Journal of Affective Disorders (128(1-2): 24-32). Recuperado de https://www.ncbi.nlm.nih.gov/ pubmed/20580435

Conway, B. R. \& Rehding, A. (2013). "Neuroaesthetics and the Trouble with Beauty". Plos Biology (11(3): e1001504). Recuperado de https://www. ncbi.nlm.nih.gov/pmc/articles/PMC3601993/

Cotrina, D. (2016). "Una vida para el arte. El pintor indigenista cajamarquino”. En Cotrina, León, Vigo \& Armas (Producción) 10 Décadas Fecundas. Andrés Zevallos de la Puente (pp. 12-13). Catálogo de exposición en el Conjunto Monumental de Belén, Cajamarca.
Csikszentmihalyi, M. (2007). Aprender a fluir. Barcelona: Kairós.

Chaterjee, A. (2011). "Neuroaesthetics: a coming of age story". Journal of cognitive neuroscience (23(1): 53-62). Recuperado de https://www.ncbi. nlm.nih.gov/pubmed/20175677

Denton, D. (2009). El despertar de la consciencia: la neurociencia de las emociones primarias. Barcelona: Paidós.

Faye, C., Mcgowan, J. C., Denny C. A. \& David, D. J. (2017). Neurobiological mechanisms of stress resilience and implications for the aged population. $\mathrm{Cu}-$ rrent neuropharmacology. Recuperado de https:// www.ncbi.nlm.nih.gov/pubmed/28820053

Fontán del Junco, M. (1996). “El lugar del artista en la sociedad contemporánea". En Ruiz, E. A. (Coord.). El oficio del artista. Sobre el concepto, situación y perspectivas de los artistas plásticos en las sociedades contemporáneas (pp. 29-64). Junta de Castilla y León. Getafe, Madrid: IMPRESA.

Frick, K. M. \& Benoit, J. D. (2010). "Use it or lose it: environmental enrichment as a means to promote successful cognitive aging". The Scientific World Journal (10: 1129-41). Recuperado de https://www.ncbi.nlm.nih.gov/pmc/articles/ PMC4462190/

Frost, R. (1934). Two tramps in mud time (read by Tom O'Bedlam). Recuperado de https://www.youtube.com/watch?v=oRosY_jyXfs

Gazzaniga, M. S. (2012). ¿Quién manda aquí? El libre albedrío y la ciencia del cerebro. Fuenlabrada: Paidós.

Gibran, K. (2010). Khalil Gibran. Una antología ilustrada. Ayman A. El-Desouky (Coord.). Barcelona: Blume.

Gogh, V. van (2004). Cartas a Theo. Barcelona: Paidós Ibérica.

Goleman, D. (2013). Focus. Desarrollar la atención para alcanzar la excelencia. Barcelona: Kairós.

Haber, S. N. (2011). "Neuroanatomy of Reward: A View from the Ventral Striatum". En Gottfried, J.A. (Editor). Neurobiology of Sensation and Reward. Frontiers in Neuroscience. (Chapter 11). Department of Neurology Northwestern University, Feinberg School of Medicine, Chicago, Illinois. Boca Raton (FL): CRC Press/Taylor \& Francis. Recuperado de https://www.ncbi.nlm.nih.gov/ books/NBK92777/\#ch11_sec2

Harari, Y. N. (2011). Sapiens. A Brief History of Humankind. London: Vintage.

Harari, Y.N. (2015). A Brief History of Tomorrow. London: Vintage. 
Jacobs, R. H. A. H., Renken, R. \& Cornelissen, F. W. (2012). Neural Correlates of Visual Aesthetics-Beauty as the Coalescence of Stimulus and Internal State (Plos one. 7(2): e31248). Recuperado de https://www.ncbi.nlm.nih.gov/pmc/ articles/PMC3285156/

Kris, E. \& Kurz, O. (1982). La leyenda del artista. Madrid: Cátedra.

Leonardini, N. (2003). El grabado en el Perú republicano. Diccionario histórico. Lima: Fondo Editorial Universidad Nacional Mayor de San Marcos.

Marin, M. M. (2015). "Crossing boundaries: toward a general model of neuroaesthetics". Frontiers in Human Neuroscience (9: 443). Recuperado de https://www.ncbi.nlm.nih.gov/pmc/articles/ PMC4528177/

MoraC. M. del S. (2014). "Reflexiones sobre neuroestética, arte e investigación". Revista Illapa Mana Tukukuq (N. 11 , pp. 68-83).

MoraC. M. del S. (2015). Creatividad \& Melancolía: Un estudio psicobiológico de las dimensiones de la personalidad en el artista plástico contemporáneo (Tesis doctoral). Departamento de Dibujo I, Facultad de Bellas Artes, UCM. Madrid: Asmat \& MoraC.

MoraC. M. del S. (2017). "De mitos y de ritos. La celebración del misterio mediante la forma y el color en el arte". En: Segura, Luza \& Asmat Chirinos-Zavala (Coordinadores) Sincretismo. Arte peruano contemporáneo (pp. 8-10). Palacio Barrantes-Cervantes. Trujillo, Cáceres: Fundación Obra Pía de los Pizarro.

Maslow, A. (2003). El hombre autorrealizado. Hacia una psicología del Ser (15. ${ }^{\text {a ed.). }}$. Barcelona: Kairós.

Munar, E., Nadal, M., Castellanos, N. P., Flexas, A., Maestú, F., Mirasso, C. \& Cela-Conde, C. J. (2012). "Aesthetic appreciation: event-related field and time-frequency analyses". Frontiers in Human Neuroscience (5: 185). Recuperado de https://www.ncbi.nlm.nih.gov/pmc/articles/ PMC3251833/

Muntane, M. D. (1994). "Biología de la creatividad”. LÁPIZ. Revista Internacional de Arte (12(104): pp. 34-41).

Pearce, M. T., Zaidel, D. W., Vartanian, O., Skov, M., Leder, H., Chatterjee, A. \& Nadal, M. (2016). "Neuroaesthetics: The Cognitive Neuroscience of Aesthetic Experience". Perspectives on psychological science (11(2): 265-79). Recuperado de https:// www.ncbi.nlm.nih.gov/pubmed/26993278

Purves, D., Augustine, G. J., Fitzpatrick, D., Katz, L. C., La Mantia, A-S. \& McNamara, J. O. (Editores) (1997). Neurosciencia. Sunderland: Sinauer Associates.
Regidor García, J. (2010). "Plástico”. En: De Felipe y González (Coordinadores). El cerebro. La gran cepa azul. Arte y neurociencia (pp. 19-29). Museo Elder de la Ciencia y la Tecnología. Las Palmas de Gran Canaria: Linca.

Rojas-Marcos, L. (2011). Superar la adversidad. El poder de la resiliencia. Madrid: Espasa Libros.

Román, E. (2005-2006). Andrés Zevallos. La utopía arcaica. Cuatro trípticos y una celebración en homenaje a José María Arguedas y Mario Urteaga. Exposición pictórica itinerante: Trujillo, Chiclayo, Cajamarca.

Román, E. (2016). "Hace 10 años conocí a Andrés. Al hombre y al pintor (29 de octubre de 1988)". En Cotrina, León, Vigo \& Armas (Producción). 10 Décadas Fecundas. Andrés Zevallos de la Puente (pp. 6-7). Catálogo de exposición en el Conjunto Monumental de Belén, Cajamarca.

Root-Bernstein, R. \& M. (2002). El secreto de la creatividad. Barcelona: Editorial Kairós.

Salas, L. (2016). "100 navidades de fecunda existencia”. En Cotrina, León, Vigo \& Armas (Producción). 10 Décadas Fecundas. Andrés Zevallos de la Puente (pp. 8-9). Catálogo de exposición en el Conjunto Monumental de Belén, Cajamarca.

Squillace, M., Picón, J. y Schmidt, V. (2011). "El concepto de impulsividad y su ubicación en las teorías psicobiológicas de la personalidad". En Revista Neuropsicología Latinoamericana (3(1) 8-18).

Stastny, F. (1967). Breve historia del arte en el Perú. Lima: Editorial Universo.

Syed, S. A. \& Nemeroff, Ch. B. (2017). Early Life Stress, Mood, and Anxiety Disorders. Chronic Stress (Thousand Oaks) 1:10. Recuperado de https://www.ncbi.nlm.nih.gov/pmc/articles/ PMC5482282/

Viosca Ros, J. (2017). El cerebro. Descifrar y potenciar nuestro órgano más complejo. México: RBA Editores.

Zeki, S. (2005). Visión interior: Una investigación sobre el arte y el cerebro. Madrid: Machado Libros.

Zeki, S. (2009). Splendors and miseries of the brain. Love, creativity, and the quest for human happiness. Oxford: Wiley-Blackwell.

Zevallos, A. (1991). Tres pintores cajamarquinos. Mario Urteaga, José Sabogal, Camilo Blas. Lima: Asociación Editora Cajamarca.

Recibido el 1 de octubre del 2017 Aprobado el 15 de octubre del 2017 